\title{
Endocrine aspects of the clinical management of breast cancer - current issues
}

\author{
$D K$ Wyld, J D Chester and T J Perren \\ ICRF Cancer Medicine Research Unit, St James's University Hospital, Beckett Street, Leeds LS9 7TF, UK \\ (Requests for offprints should be addressed to T J Perren)
}

\section{Introduction}

Endocrine therapy has now been used as an effective treatment for breast cancer for 100 years. It was the first successful systemic treatment for cancer, initially becoming accepted following George Beatson's (1896) observations that, in a proportion of pre-menopausal women with advanced breast cancer, bilateral oophorectomy resulted in disease regression. Over the subsequent 75 years, several other endocrine therapies for breast cancer were developed, including other surgical approaches to hormone ablation therapy such as adrenalectomy (Huggins \& Dao 1953) and hypophysectomy (Luft \& Olivecrona 1953), and hormonal additive therapies, such as the use of pharmacological doses of androgens, oestrogens, progestogens and glucocorticoids. However, as none of these approaches led to a significant improvement in the rates of tumour regression, clinicians' enthusiasm in the 1960s and early 1970s became focused on the use of newly developing cytotoxic chemotherapy regimens. Then, in the $1970 \mathrm{~s}$, tamoxifen, and subsequently a range of other new endocrine agents, became available which were of low toxicity and generally well tolerated - high-dose oestrogens were quickly replaced by tamoxifen, and adrenalectomy by aromatase inhibitors. Measurement of hormone receptor levels also became available, allowing better selection of patients whose tumours might be hormonally responsive. In addition, some of the limitations of cytotoxic drugs in breast cancer were starting to become apparent.

Randomised trials with these new agents, and in particular with tamoxifen, clearly demonstrated the benefit of endocrine therapy in both the adjuvant and metastatic disease settings. The use of such treatments has substantially increased over the last two decades, to the extent that virtually all patients with breast cancer can now be expected to receive at least one form of endocrine manipulation at some point during the course of their treatment.
Despite this progress, there is clearly a need for further improvements in the management of patients with breast cancer in general, and endocrine therapy in particular.

\section{Scope of this review}

Endocrine therapies in breast cancer, including mechanisms of action, detailed pharmacology, and the current status of new therapies have been extensively reviewed (Stein et al. 1995, Howell et al. 1996b). The aim of this review is to discuss a number of topical clinical issues in the endocrine treatment of breast cancer. In particular, we will focus on current issues surrounding the use of tamoxifen in routine practice such as the risk of second malignancies, optimal duration of therapy, and toxicity in long-term treatment. We will also discuss the role of ovarian suppression, and go on to consider new aromatase inhibitors, and combinations of different treatment modalities in the therapy of breast cancer, and the use of hormone replacement therapy in those women with a previous diagnosis of breast cancer. On-going clinical trials of endocrine therapies in breast cancer will also be discussed.

\section{Established endocrine therapies}

\section{Hormonal manipulation: tamoxifen}

Tamoxifen is of proven benefit in the clinical management of patients with breast cancer, both in the adjuvant and metastatic settings. As such, it is the most widely prescribed of the anticancer agents, with at least a million women world-wide taking the drug. The optimal use of tamoxifen in clinical practice, with maximal benefit and minimal toxicity, is still an area of intense investigation, particularly in the adjuvant setting. A large amount of new information has become available recently, answering some questions but also, in some instances, provoking considerable debate. 


\section{Wyld et al.: Endocrine aspects of the clinical management of breast cancer}

The latest up-dated overview of tamoxifen in clinical trials has recently been published. The 1998 Early Breast Cancer Trialists' Collaborative Group (EBCTCG) overview analyses data up to 1995 on 36689 women in 55 trials commenced before 1990, comparing tamoxifen treatment versus no tamoxifen - estimated to be about $87 \%$ of the total world-wide evidence. As in the previous, 1992, overview significant reductions in tumour recurrence and in mortality were observed in almost all breast cancer patients: both axillary node-negative and node-positive, and irrespective of age, menopausal status, tamoxifen dose and whether adjuvant chemotherapy had been given. Once again there was a significant trend towards greater benefits from a longer duration of tamoxifen therapy. The previous observations of a reduction in the occurrence of contra-lateral breast cancer, now quantified as being twice as large as the increase in risk of endometrial cancer in tamoxifen-treated patients, were also confirmed. A significant development since the previous overview, however, was a clarification of the importance of oestrogen receptor (ER) status in predicting response to tamoxifen. In the $21 \%$ of women studied who had low or zero levels of ER in pathological specimens, the effects of tamoxifen were considerably smaller than in women who had positive, or unknown, ER status.

\section{Optimal duration of therapy}

Probably the most controversial issue over recent years has been, and continues to be, the question of the optimal duration of adjuvant tamoxifen therapy. The EBCTCG $(1992 a, b)$ overview addressed the issue of tamoxifen duration. The scheduled duration of tamoxifen in the trials analysed varied between 6 months and 5 years, with few trials that directly compared longer with shorter treatment. Conclusions were therefore mainly based on indirect comparisons of results from trials of various durations of tamoxifen treatment versus no treatment. These analyses showed that a period of 2-5 years of tamoxifen therapy was significantly more effective in delaying recurrence and increasing survival than a shorter duration.

The Swedish Breast Cancer Co-operative Group trial (SBCCG 1996), randomised 3887 post-menopausal women to receive tamoxifen for either 2 or 5 years. At a median follow-up of 5.5 years, there was a statistically significant improvement in both event-free and overall survival in the group which received 5 years of tamoxifen treatment. The benefit associated with the longer treatment extended to women with lymph node-positive as well as lymph node-negative disease, but it appeared to be restricted to women whose tumours were classified as ER positive.

Further support for 5 years' tamoxifen treatment, rather than 2, comes from preliminary data from the Current Trials Working Party of the Cancer Research
Campaign's Breast Cancer Trials Group (1996). Amongst 2937 patients randomised to either stop tamoxifen after 2 years, or to continue to complete 5 years, there was no significant difference in survival at a median follow-up of 2 years, but there was a significant delay in time to relapse in those patients amongst the group randomised to continue tamoxifen therapy to 5 years who had actually completed the full 5 years of tamoxifen at the time of the preliminary analysis.

Further support for longer duration of tamoxifen therapy comes from the EBCTCG 1998 review. Statistically significant trends in reductions in recurrence (21, 29 and $47 \%$ for 1, 2 and 'about 5' years on tamoxifen respectively) and mortality (12, 17 and $26 \%$ respectively) were seen with increasing duration of therapy, although the authors are careful to point out that, in their analysis, such comparisons are only indirect, as few of the trials analysed were designed specifically to address the optimal duration of tamoxifen therapy.

From these data, it seems clear that 5 years of tamoxifen therapy is superior to 2 years. However, it remains uncertain whether continuing tamoxifen beyond 5 years provides any further benefit. The Scottish Tamoxifen trial (Stewart et al. 1996) studied 342 patients with operable breast cancer, randomised at the 5-year point to either stop tamoxifen or to continue for a further 5 years. Median follow-up was for up to 11 years after the commencement of tamoxifen therapy. There were non-significant differences between the two study groups in relapse (with the trend towards relapse being greater in the group continuing tamoxifen: 38 versus 28\%), disease-free survival and incidence of endometrial carcinoma (which was also slightly greater in the group continuing tamoxifen). This study therefore concluded that if continuing tamoxifen beyond 5 years is beneficial, the extent of that benefit is relatively modest, and not comparable with the benefits seen in the first 5 years of treatment, and that until more trial data are available, 'there is little to suggest that tamoxifen should be prescribed routinely beyond 5 years'.

The National Surgical Adjuvant Breast and Bowel Project (NSABP) B-14 study of adjuvant tamoxifen in operable primary breast cancer was performed in two phases. The first phase had demonstrated substantial benefits of tamoxifen therapy in breast cancer patients with early stage disease (Fisher et al. 1989, Fisher \& Redmond 1992). The second phase, investigating discontinuation of tamoxifen therapy after 5 years versus continuation for a further 5 years, to a total of 10 years was, however, terminated when findings from interim analyses indicated no additional benefit from tamoxifen therapy beyond 5 years. Whilst there was no significant difference in survival between those continuing tamoxifen to 10 years and those in whom it was discontinued after 
5 years $(96 \%$ versus $94 \% ; P=0.08)$, there were statistically significant improvements in disease-free $(92 \%$ versus $86 \% ; P=0.003)$ and distant disease-free survival $(96 \%$ versus $90 \% ; P=0.01)$ in the group in whom tamoxifen was discontinued after 5 years (Fisher et al. 1996).

On the basis of the (at that point, unpublished) results of the Scottish and NSABP B-14 trials, the National Cancer Institute (NCI) released a clinical announcement, which stated that these results provided no evidence of benefit for continuing tamoxifen beyond 5 years, and that both trials suggest a greater likelihood of relapse in women who take tamoxifen for greater than 5 years when compared with women who receive 5 years of therapy. They concluded that 'although the NSABP B-14 trial and the Scottish trials provide evidence that continuation beyond 5 years is not beneficial, the optimal duration of adjuvant tamoxifen treatment still remains to be determined' and that while awaiting results of further trials, 'all available evidence indicates that 5 years of tamoxifen is a reasonable standard for the adjuvant setting' (National Cancer Institute 1995). This announcement sparked considerable discussion and debate at the time.

However, following the NCI announcement, the Eastern Co-operative Oncology Group (ECOG) published their results from a randomised trial performed to assess the efficacy of maintaining tamoxifen therapy beyond 5 years in 194 women with axillary lymph node-positive breast cancer who had been treated with surgery followed by 1 year of chemotherapy and 5 years of tamoxifen (Tormey et al. 1996). No statistically significant differences were noted in either time to relapse or survival between women continuing tamoxifen and those stopping after 5 years. The size of this study means that it does not have sufficient power to detect small differences in outcome, but, clearly, the equivalent relapse-free survival figures contrast with the NSABP B-14 findings. There is, therefore, as yet, insufficient data to answer the question regarding therapy for longer than 5 years.

Although longer follow-up of the Scottish, NSABP B-14 and ECOG trials may help, in part, to answer the question, what is needed is further trials of large numbers of women, randomly assigned to different tamoxifen durations (Peto 1996). Clinicians are, therefore, being encouraged to enter patients into two large, ongoing international studies.

The Adjuvant Tamoxifen: Longer Against Shorter (ATLAS) trial randomly assigns patients who have any duration of tamoxifen treatment (with 2 years being recommended but not essential) to either stop tamoxifen or to receive it for a further 5 years. A related trial, the Adjuvant Tamoxifen Treatment - Offer More? (aTTom) trial recruits post-menopausal patients with breast cancer who have received 2 or more years of tamoxifen therapy and randomly assigns them to either stop tamoxifen treatment or to continue it for a further 3 years. Recruitment targets of 8000 women in the UK (in aTTom) and 20000 world-wide (in collaboration with the ATLAS trial) are intended to generate sufficiently powerful data reliably to answer the question as to the optimum duration of tamoxifen therapy. Between October 1995 and October 1997, 2456 women have been randomised within the aTTom trial, at 94 centres in the UK, towards the target of 8000. It will, therefore, be several years before we can provide any definite answers to the question of whether more than 5 years of adjuvant tamoxifen treatment will result in better long-term survival. However, the question must be answered reliably, as even a small benefit in a disease as common as breast cancer could lead to a substantial number of lives being saved.

\section{Risk of second malignancies with tamoxifen use}

The carcinogenic potential of tamoxifen has caused considerable concern. It remains an important issue, as its widespread usage means that even a very small increased percentage risk would affect a large number of women, including women with early disease and, hence, high cure rates. Healthy women in chemoprevention trials (see below) would also be at risk.

Tamoxifen has been shown to be carcinogenic in rats (Greaves et al. 1993, Hard et al. 1993, Williams et al. 1993), with high doses shown to produce liver tumours. In humans, there is now an accepted association between tamoxifen use and an increased risk of endometrial cancer, but as yet no definite association with an increased risk of any other malignancies (Jordan 1995b, Barakat 1996).

Endometrial carcinoma In the mid 1980s, a number of authors reported the occurrence of endometrial cancers in patients with breast cancer receiving tamoxifen (Killackey et al. 1985). The strongest data initially linking tamoxifen use and the subsequent development of endometrial cancer was the Stockholm trial (Fornander et al. 1989). They found a relative risk of endometrial cancer of 6.4 in 931 tamoxifen-treated patients compared with 915 controls. In the NSABP B-14 study (Fisher et al. 1994), there were 23 cases of endometrial cancer in the tamoxifentreated groups, compared with 2 in the placebo group. However, the authors concluded that the benefit of tamoxifen therapy for breast cancer outweighed the potential increase in endometrial cancer.

An analysis of the combined data from three large Scandinavian breast cancer trials (the Stockholm trial, the Danish Breast Cancer Group Trial and the South-Swedish Trial) has confirmed the relationship between tamoxifen use and the development of endometrial cancer. These studies included a total of 4914 patients with a median follow-up of 8-9 years (Rutqvist et al. 1995). A statistically significant increase in endometrial cancers 
among tamoxifen-treated patients was found, with a relative risk of 4.1 .

A highly statistically significant increase in the incidence of endometrial carcinoma in women taking tamoxifen was also seen in the EBCTCG 1998 review. On the basis of the 124 cases of endometrial cancer occurring in women in the trials analysed, they estimate a doubling of the risk of endometrial cancer in women taking tamoxifen for 1 or 2 years, and a quadrupling of the risk in women on about 5 years of tamoxifen. They do stress, however, that this is outweighed by a reduction in the incidence of contra-lateral breast cancer in women taking tamoxifen which is approximately double the increase in endometrial cancer.

Assikis et al., (1996) have reviewed the world literature between 1984 and 1995 regarding tamoxifen and endometrial cancer, including case reports, case control studies and randomised trials in which the issue of second malignancy was addressed prospectively. They found reports of 349 endometrial carcinomas in women taking tamoxifen for breast cancer. They estimate a definite, low increase of two- to threefold in the risk of endometrial cancer in women taking tamoxifen, but point out that the publicity surrounding the association between endometrial cancer and tamoxifen use may produce a selection bias, with treated patients being more likely to be investigated by a gynaecologist.

The link between tamoxifen use and the development of endometrial cancer is, therefore, now widely accepted. Several other factors are less clear. With respect to possible pathogenesis, it is known that oestrogen use increases the incidence of endometrial cancer in postmenopausal women (relative risk $=2.3$ for oestrogen users versus non-users, Grady et al. 1995), and that tamoxifen therapy increases the likelihood of finding oestrogen-like effects in the uterus. Therefore, it is likely that the oestrogenic effects of tamoxifen on the uterus contribute to its carcinogenic potential. This raises the possibility that this risk may be avoided by the use of new 'pure' antioestrogens (see Table 1 and 'Emerging endocrine therapies' below).

Table 1 Endocrine therapies for breast cancer: past and present.

\begin{tabular}{|c|c|}
\hline \multicolumn{2}{|l|}{ Endocrine gland ablation/suppression } \\
\hline Oophorectomy & $\begin{array}{l}\text { Surgery } \\
\text { Radiotherapy }\end{array}$ \\
\hline Adrenalectomy & Surgery \\
\hline Hypophysectomy & Surgery \\
\hline \multicolumn{2}{|l|}{ Hormone manipulation } \\
\hline Progestogens & $\begin{array}{l}\text { Medroxyprogesterone acetate } \\
\text { Megestrol acetate }\end{array}$ \\
\hline \multicolumn{2}{|l|}{ Anti-oestrogens } \\
\hline Non-steroidal & $\begin{array}{l}\text { Tamoxifen } \\
\text { Toremifene } \\
\text { Raloxifene }\end{array}$ \\
\hline 'Pure', steroidal anti-oestrogens & Faslodex (ICI 182780$)$ \\
\hline $\begin{array}{l}\text { Aromatase inhibitors } \\
\text { Non-steroidal }\end{array}$ & $\begin{array}{l}\text { Aminogluthethimide } \\
\text { Fadrozole } \\
\text { Anastrozole } \\
\text { Letrozole }\end{array}$ \\
\hline Steroidal & $\begin{array}{l}\text { 4-Hydroxy-androstenedione } \\
\text { Exemestane }\end{array}$ \\
\hline GnRH analogues & $\begin{array}{l}\text { Goserelin } \\
\text { Leuprorelin }\end{array}$ \\
\hline Anti-progestogens & $\begin{array}{l}\text { Mifepristone } \\
\text { Onapristone }\end{array}$ \\
\hline
\end{tabular}

$\mathrm{GnRH}$, gonadotrophin releasing hormone. 
Gastrointestinal tumours The finding of an increase in liver tumours in rats that received tamoxifen (albeit at doses 20-100 times those used in humans) and a report of 2 liver tumours in 931 patients who received $40 \mathrm{mg}$ tamoxifen per day compared with no tumours in 915 control patients (Fornander et al. 1989) caused considerable concern regarding the risk of development of such tumours in tamoxifen-treated women. However, on the basis of the results from a number of subsequent studies which have shown no evidence that tamoxifen use is associated with an increased incidence of liver tumours, the evidence would appear to suggest that, in fact, this is neither clinically nor biochemically relevant (Jordan 1995a).

Both the Stockholm trial (Rutqvist et al. 1993), and joint analysis of the three Scandinavian groups referred to above (Rutqvist et al. 1995) showed an excess of gastrointestinal tumours in tamoxifen-treated patients. Most of this excess involved colorectal cancers and stomach cancer. There was no substantial increase in any other type of gastrointestinal cancer (including liver cancer). However, much doubt has subsequently been cast on the reliability of this data and on the analytical methods used (Jordan 1995a, Simon 1995). Several other studies, including the NSABP B-14 trial (Fisher et al. 1994, 1996) did not demonstrate a significant increase in second tumours at any site other than the endometrium.

Other malignancies: the Surveillance, Epidemiology and End-Results programme On a larger scale, the risk of second cancers among 87323 women with breast cancer has been evaluated in the Surveillance, Epidemiology and End Results (SEER) programme (Curtis et al. 1996). The results of this study are important, in that they are derived from a very large cohort of patients and are in keeping with results obtained from smaller trial data. However, it should be noted that, in the SEER data, there is a significant lack of detailed information on individual patients' initial or subsequent treatment. As a result, the 'tamoxifen group' represents a subgroup of women considered most likely to have received tamoxifen as part of their initial therapy for early stage disease. The authors have estimated that $90-95 \%$ of the 'tamoxifen group' were actually treated with the drug. Also, few women were followed-up for more than 10 years, and there is an absence of data on other risk factors for second tumours (e.g. oestrogen use). Noting these limitations, patients with breast cancer initially treated with tamoxifen did develop second cancers at a slightly higher rate than expected in the general population, but identical to that experienced by breast cancer patients with no tamoxifen or unknown therapy.

Interestingly, the SEER programme demonstrated a decreased risk of contra-lateral breast cancer in the tamoxifen group versus the no tamoxifen/unknown group.
The 1998 EBCTCG overview has confirmed and quantified this, with proportional reductions in the incidence of contra-lateral breast cancer of a massive $47 \%$ in women taking tamoxifen for 5 years. Such data support the conclusion that tamoxifen might have a role in breast cancer prophylaxis (see below).

The SEER study confirmed the increased incidence of endometrial carcinoma in patients treated with tamoxifen. The actuarial risk of uterine cancer among postmenopausal women after adjuvant tamoxifen was $1.8 \%$ at 10 years, compared with $0.9 \%$ in the general population. However, cancers of the colon, rectum, and stomach were not in excess following tamoxifen therapy. Again, support comes from the 1998 EBCTCG overview, which showed no apparent effect on the incidence of any other cancer, other than endometrial and contra-lateral breast cancers, and including colorectal carcinoma.

\section{Other long-term treatment issues}

In breast tissue, tamoxifen has a predominantly antioestrogenic role. In most other tissues, however, its properties are mostly agonist, oestrogenic effects. As in the case of oestrogenic hormone replacement therapy (HRT), tamoxifen has been shown to have both beneficial and detrimental effects on general health in postmenopausal women via its agonist effects in non-breast tissues. These may have particular significance in its use in the adjuvant setting or in chemo-prevention trials.

Cardiovascular disease risk Conflicting data has come from the trials of adjuvant therapy for early breast cancer regarding a possible effect on the rate of cardiovascular deaths for women receiving tamoxifen. Most trials, including the NSABP B-14 trial (Fisher et al. 1996) have not reported a significant difference in the death rate from cardiovascular disease between tamoxifen-treated groups and controls. However, the Scottish adjuvant tamoxifen trial (McDonald \& Stewart 1991) reported a significant reduction in the incidence of fatal myocardial infarction in post-menopausal women receiving tamoxifen as compared with control patients. A subsequent cohort study linked this trials database and statistics of Scottish hospital in-patients to identify reports of cardiac and vascular morbidity. In this analysis, tamoxifen use was also associated with lower rates of myocardial infarction (McDonald et al. 1995).

Similar results were seen in the Stockholm tamoxifen trial (Rutqvist et al. 1993), but the reduction in cardiac deaths did not reach statistical significance because of the small number of deaths occurring in their sample.

A potential mechanism for any putative protective effect for tamoxifen in cardiovascular disease may be suggested by two studies (Love et al. 1990, Powles 1996) showing alterations in cholesterol metabolism, producing 
reduced low density lipoprotein:high density lipoprotein ratios.

Bone mineral density With both oestrogenic and antioestrogenic effects, tamoxifen has the potential to influence bone turnover. As with hormone replacement therapy, agonist activity can potentially protect against bone loss. However, anti-oestrogenic action can result in bone mineral loss and possibly, therefore, increase the risk of osteoporosis. The exact effect of tamoxifen on bone mineral density (BMD) has not yet been clearly defined, particularly with regard to the long-term risks or benefits. For now, it remains unclear whether tamoxifen has any effect on fracture rates during long-term follow-up. There has also been very little evaluation of its effects in premenopausal women.

Relatively recent data have suggested that adjuvant tamoxifen, at doses of $20-30 \mathrm{mg} /$ day over 2 years, is associated with an increase in BMD in the lumbar spine (Love et al. 1988, Kristensen et al. 1994). However, the effect may be different in pre- and post-menopausal women. As part of a placebo-controlled tamoxifen chemoprevention trial (Powles et al. 1996), BMD in the lumbar spine and hip was evaluated using dual-energy-X-ray absorptiometry (DEXA). It suggested contrasting effects of tamoxifen in healthy pre-menopausal and post-menopausal women. Tamoxifen was shown to cause a reduction in BMD in pre-menopausal women, but an increase in post-menopausal patients. Most of these changes occurred in the first and second year of tamoxifen usage, and by the third year there seemed to be some stabilisation of BMD.

Thrombo-embolic disease Recent data from adjuvant trials have confirmed the previously noted increased incidence of thrombo-embolic events in patients treated with tamoxifen compared with controls, but suggested that the overall incidence of such events remains relatively low. A retrospective review of the Scottish trial data (McDonald et al. 1995) reported that hospital admissions for deep venous thrombosis and pulmonary embolus were increased for patients allocated tamoxifen compared with controls, and this is significant when current users are compared with non-users. Similar findings came from the NSABP B-14 study (Fisher et al. 1996), in which embolic phenomena, deep venous thrombosis and hospitalisation all occurred more often in the tamoxifen-treated group than in the placebo group.

Ocular effects of tamoxifen Tamoxifen is known to have the potential to cause a range of ocular damage, including corneal changes, cataracts and retinopathy. However, a recent review (Nayfield \& Gorin 1996) concluded that ocular toxicity is uncommon in the current clinical setting of long-term, low-dose tamoxifen use.

\section{Summary of long-term treatment issues}

Overall, recent findings have led to a general acceptance of an association between tamoxifen use in humans and an increased risk of endometrial cancer, although several important issues regarding this increased risk need further clarification. There is also a definite reduction in the incidence of contra-lateral breast cancer associated with tamoxifen use, which appears to outweigh the increased risk of endometrial cancer. At the present time there is no definite association with an increased risk of any other malignancy. Peto (1996) has stated 'there is no good evidence that any causes of death other than breast cancer or endometrial cancer are affected by tamoxifen'. The 1998 EBCTCG overview concurs: 'Tamoxifen had no apparent effect.., after exclusion of deaths from breast or endometrial cancer, on any of the other main categories of cause of death'. What is clear is that, when the potential benefits of its oestrogen agonist properties, such as an improved lipid profile and increased bone mineral density, are considered, the benefits of tamoxifen therapy in breast cancer patients far outweigh any risks associated with the treatment, including the risks of second cancers.

\section{Tamoxifen in chemo-prevention trials}

Several trials are under way in Europe and North America where tamoxifen is administered to patients who do not have breast cancer, but who have a family history of the disease, in the hope of avoiding subsequent development of the disease. The rationale for this is based primarily on the combined evidence from eight trials (reviewed in Nayfield et al. 1991), and confirmed by results of the SEER study mentioned above, that women with breast cancer and treated with adjuvant tamoxifen have a 35\% reduction in the risk of subsequently developing breast cancer in the contra-lateral breast. Furthermore, trials of tamoxifen in an adjuvant setting have demonstrated that it is better to give tamoxifen early, rather than waiting for disease recurrence (Breast Cancer Trials Committee: The Scottish Trial 1987).

In April 1998, only 7 months after completing recruitment, the National Cancer Institute of the USA released data on the internet on the 13388 high-risk women participating in the NSABP's Breast Cancer Prevention Trial (BCPT), 14 months earlier than scheduled. At a maximum follow-up of six years, there were significantly fewer incidences of new breast cancer in the tamoxifen group (85 cases), compared with the placebo control group (154 cases) - a reduction of $45 \%$. Similar reductions were seen in all age groups. There were also fewer diagnoses of ductal carcinoma in situ (DCIS). Increased incidence of endometrial carcinoma (33 versus 14) and thromboembolic disease (47 versus 25 ) were also seen in the tamoxifen group. To date, there has been no significant 
difference in the incidence of myocardial infarction, but there has been a reduction in the number of bone fractures. In view of the magnitude of this result, the monitoring committe felt ethically obliged to release data, so that those taking the placebo could 'cross-over' to tamoxifen and gain its apparent benefits.

Controversy has followed the announcement, as the data are immature, with only a relatively short period of follow-up so far. For this reason, it has been decided that the parallel International Breast Cancer Intervention Study (IBIS), whose design is similar to the NSABP BCPT, should continue recruiting as planned. As of 1st April 1998, the trial had recruited 4500 out of a planned 7000 patients, and recruitment is expected to be completed around the year 2000 .

If the IBIS trial confirms the initial suggestions from the BCPT, then this, of course, raises the question as to whether this is sufficient to justify the risks of tamoxifen toxicity in the many women receiving tamoxifen who would not develop breast cancer.

There are various ways around this ethical dilemma. The strategy adopted by the BCPT and IBIS trials is not to treat all healthy women, but to target women at particularly high-risk of breast cancer, such as post-menopausal women with a strong family history of breast cancer. Another is the development of other, new anti-oestrogens with a better toxicity profile than tamoxifen (see below).

\section{Ovarian suppression}

The role of ovarian ablation in the adjuvant setting was first evaluated in randomised trials in the 1950s, but it took until the early 1990s before its potential importance in routine clinical practice became clear. In the EBCTCG's first overview (1990), ovarian ablation data were incomplete, and the results of several small trials were inconsistent. The emphasis in adjuvant therapy was, therefore, on tamoxifen and chemotherapy. However, in the EBCTCG's 1992 overview, a meta-analysis of 4000 women in 12 trials which had commenced before 1985, a clear role for ovarian suppression in the adjuvant therapy of pre-menopausal women with breast cancer became clear.

The 1992 review showed that, in 1746 women under the age of 50 years, ovarian ablation resulted in a $26 \%$ reduction in the annual rate of recurrence and a $25 \%$ decrease in the annual rate of death. Ovarian ablation in this group produced significant mortality reductions both during treatment and up to 9 years of follow-up. The effect of ovarian suppression appeared to be somewhat smaller in the presence than in the absence of cytotoxic chemotherapy, but this difference was not statistically significant, and even in the presence of cytotoxic chemotherapy ovarian ablation still significantly reduced both recurrence and mortality. Conversely, for 1326 women over the age of 50 ovarian ablation had no significant effect on either recurrence-free or overall survival.

The EBCTCG has subsequently published its updated overview of the randomised trials of ovarian ablation in early breast cancer, after 15 years follow-up (EBCTCG 1996). This now includes data from 12 of the 13 studies that assessed ovarian ablation by irradiation or surgery, all of which began before 1980 (four studies which assessed ovarian suppression by drugs, all of which began after 1985, were not included in the analysis). Among 2102 women aged under 50 at the time of randomisation, 15year survival and recurrence-free survival were significantly improved among those allocated ovarian ablation. In the 1354 women aged 50 or over when randomised, similar trends were seen, but these did not reach statistical significance.

Although the 1992 overview showed a similar benefit for chemotherapy and ovarian ablation separately, these effects did not appear additive. In the 1996 review, the risk reduction produced by ovarian ablation appeared to be smaller in the presence of additional cytotoxic chemotherapy (i.e. in patients who received ablation plus chemotherapy rather than chemotherapy alone), possibly because (at least part of) the benefit of chemotherapy might be via suppression of ovarian function. However, the numbers involved in making such indirect comparisons were acknowledged by the authors to be too small to be statistically reliable.

The authors of the 1996 overview concluded that ovarian ablation significantly improved long-term survival in women less than 50 years of age, at least in the absence of chemotherapy, but that further trials' data were necessary to establish the additional benefits of ovarian ablation when used in combination with other adjuvant treatment modalities.

The exact role of ovarian ablation in the routine clinical management of women less than 50 years old thus remains unclear, despite the results of the 1996 overview. Further information will be available over the next few years which should assist in defining the role of ovarian ablation/suppression in the adjuvant therapy of early breast cancer. Longer follow-up of trials used for the 1996 EBCTCG overview, as well as results from trials begun more recently will be available for the next EBCTCG overview in 2000. This will also include trials of ovarian suppression with luteinizing hormone releasing hormone agonists, including more than 3000 women. However, additional randomised trials are required to help to define further the role of ovarian ablation in the management of early breast cancer, and to address a number of still unanswered clinical questions, including comparisons of the combination of ovarian suppression and chemotherapy 
with either modality alone, and also the effects of adjuvant ovarian ablation in patients already taking tamoxifen, and the relevance of hormone receptor measurement to outcome following treatment.

One trial designed to answer some of these questions is the United Kingdom Central Co-ordinating Committee for Cancer Research (UKCCCR) adjuvant breast cancer (ABC) trial, which was launched in 1993. Within the trial all patients are treated with prolonged adjuvant tamoxifen $(5$ years is currently recommended, however patients may also be entered into trials of tamoxifen duration if felt appropriate). If uncertainty exists as to whether pre- or peri-menopausal women should receive chemotherapy and/or ovarian suppression, in addition to tamoxifen, the clinician is free to offer randomisation to one or both options - i.e. patients may be randomised between chemotherapy and no chemotherapy, or between ovarian suppression versus no ovarian suppression, or may enter both randomisations. In post-menopausal women the randomisation is between tamoxifen alone and tamoxifen plus chemotherapy. The aim is to accrue sufficient patients (approximately 2000 in each arm of the study) to detect a $5 \%$ difference in survival between arms of the study. As of 31 st October 1997, 2925 patients, out of a planned total of 6000 , had been accrued.

\section{Emerging endocrine therapies}

With the proven efficacy of tamoxifen, much attention has been focused on producing even better hormonal therapies for breast cancer. In particular, investigators have searched for agents with improved therapeutic ratio, with even better efficacy but lower toxicity, particularly a lower risk of second malignancy. Recent interest has focused on two particular types of agent: anti-oestrogens other than tamoxifen, and aromatase inhibitors.

\section{New anti-oestrogens}

There are two classes of anti-oestrogens: the non-steroidal agents, which include tamoxifen, and steroidal or 'pure' anti-oestrogens.

One randomised controlled trial of 648 postmenopausal patients with metastatic breast cancer suggests that the non-steroidal anti-oestrogen toremifine might represent an alternative to tamoxifen as a first-line endocrine agent, showing no less efficacy, as measured by objective response rate, overall survival or disease-free survival, but inducing less nausea (Hayes et al. 1995).

The most promising of the 'pure', steroidal antioestrogens is faslodex (formerly known as ICI 182 780), an oestradiol derivative which acts via a novel mechanism involving destruction of newly-synthesised oestrogen receptors and does not show cross-resistance with tamoxifen. It has been investigated as a second-line hormonal therapy in 19 post-menopausal women with metastatic breast cancer, following tamoxifen failure (Howell et al. $1996 a, b$ ), achieving a $37 \%$ partial response rate, and stable disease in a further $32 \%$. Median duration of response was 25 months, raising the possibility that faslodex may lead to longer durations of response than tamoxifen and progestogens. However, this was only a small trial, and published clinical experience of the drug is still limited.

In a review of various new endocrine therapies, Howell et al. (1996) concluded that none of the new antioestrogens showed greater efficacy than tamoxifen, while the non-steroidal type were similar in terms of toxicity, and the steroidal type slightly less toxic. The non-steroidal anti-oestrogens, with partial agonist activities similar to tamoxifen, do, however, have the potential for beneficial cardiovascular and skeletal effects.

Gradishar and Jordan (1997) have reviewed several new anti-oestrogens, and conclude that while several of these are promising, there is, thus far, insufficient clinical experience with any of the new agents to advocate any of them as a replacement of tamoxifen at present.

\section{Aromatase inhibitors}

Aromatase inhibitors are a class of compounds which are already beginning to replace progestogens as second-line endocrine therapy following tamoxifen failure in postmenopausal patients with advanced disease, and are currently undergoing trials as alternatives to tamoxifen in first-line adjuvant therapy.

The mechanism of action of the aromatase inhibitors differs from that of tamoxifen. Biosynthesis of oestrogen occurs in a process in which the male sex hormones, androgens, are converted to female sex hormones, oestrogens. In pre-menopausal women, this occurs principally in the ovaries. In post-menopausal women, however, peripheral conversion of circulating androstenedione to oestrone and subsequently to oestradiol occurs in peripheral tissues via a different pathway, involving an aromatase enzyme complex which is localised in tissues such as fat (including fatty tissues of the breast, and breast tumours), liver, muscle, and in the brain. In post-menopausal women, this aromatase enzyme complex is not affected by a feedback mechanism, and therefore its inhibition can cause a significant decrease in tissue oestrogen levels.

The first aromatase inhibitor to be used therapeutically in the management of patients with breast cancer was aminoglutethimide. Originally developed as an anticonvulsant, but found to have the adverse effect of inducing adrenal insufficiency, it was introduced as a medical alternative to surgical adrenalectomy in postmenopausal patients and shown to be as effective as surgical ablation in a randomised trial (Santen et al. 1981). Subsequent randomised trials showed it to be just as 
effective as tamoxifen in the treatment of metastatic breast cancer in post-menopausal women. Despite this demonstration of its efficacy in advanced breast cancer, it had profound side-effects including lethargy, dizziness, skin rashes, and thyroxine synthesis blockade.

In an attempt to achieve efficacy at least as good as aminoglutethimide, but without the debilitating toxicities, a number of new aromatase inhibitors with better therapeutic indices have been developed (see Table 1), and several of these have recently become available for routine clinical use. Like the new anti-oestrogens mentioned above, these are of two basic types: steroidal and nonsteroidal.

Formestane (4-hydroxyandrostenedione, 4-OHA) is a steroidal aromatase inhibitor, 30-60 times more potent than aminoglutethimide. It was the first of the second generation aromatase inhibitors to become commercially available for the treatment of patients relapsing on tamoxifen. In a randomised, controlled trial, formestane produced response rates equivalent to tamoxifen (Goss \& Gwyn 1994). However, due to its conjugation by first-pass metabolism in the liver, it requires intra-muscular injection every 2 weeks.

Of the newer aromatase inhibitors, the most widely used orally administered drug, at present, is anastrozole. It is a non-steroidal compound which combines potency (100-150 times more potent than aminoglutethimide) and high selectivity for aromatase with no discernible effects on adrenal function at the maximally effective aromatase inhibiting doses.

Two phase III studies comparing anastrozole (1 and $10 \mathrm{mg}$ once daily) with megestrol acetate (40 mg four times daily) in post-menopausal women with advanced breast cancer, and whose disease had progressed following tamoxifen treatment, have recently been conducted. These two independently conducted trials were of the same protocol design which has allowed a combined analysis of the data to be performed (Buzdar et al. 1996). The combined analysis included results from 764 randomised patients, with median duration of follow-up of approximately 6 months. Approximately one third of patients in each group benefited from treatment. Anastrozole and megestrol acetate were both well tolerated. Gastrointestinal disturbance was significantly more common among patients in the $10 \mathrm{mg}$ anastrozole dose group, but not in the $1 \mathrm{mg}$ dose group. Weight gain was significantly less with anastrozole at both dose levels than with megestrol acetate.

Updated results from this combined analysis have recently been presented at the 33rd Annual Conference of the American Society of Clinical Oncology. Significant improvement in median and two-year overall survival has been seen, comparing anastrozole $(1 \mathrm{mg})$ - and megestrol acetate-treated groups. The individual studies were consistent in each demonstrating a lower risk of death on anastrozole (both $1 \mathrm{mg}$ and $10 \mathrm{mg}$ doses) compared with megestrol acetate.

As a result of such studies, aromatase inhibitors are now beginning to replace progestogens as second-line therapy in patients who have relapsed on tamoxifen therapy. Two recently published trials (Falkson \& Falkson 1996, Thurlimann et al. 1996) have investigated the second generation aromatase inhibitor, fadrozole, not just as second-line endocrine therapy, but as an alternative to tamoxifen as first-line therapy in post-menopausal women with advanced disease (reviewed in Cocconi 1996). In the larger study (Thurlimann et al. 1996), there were equivalent (though surprisingly low, in absolute terms) response rates. Duration of response was also similar in the two treatment arms. However, tamoxifen was superior in terms of time to treatment failure (though this did not reach significance when adjustment was made for prognostic factors), while fadrozole had significantly lower toxicity. The other study (Falkson \& Falkson 1996) had slightly conflicting conclusions. Again, efficacy was similar in the two arms, but there was no significant difference in time to treatment failure or toxicity. Overall, the conclusion is that fadrozole represents an agent with good efficacy and tolerability, but it has not proved superior to tamoxifen as a first-line agent.

Randomised trials are also on-going to assess anastrozole as first-line therapy both in advanced disease, and in adjuvant therapy for early disease. The TARGET (Tamoxifen versus Arimidex Randomised Group Efficacy and Tolerability) trial compares anastrozole with tamoxifen as first-line therapy in advanced disease, where 'firstline' includes both those patients who have never received tamoxifen and those who completed tamoxifen therapy more than 12 months previously. In the adjuvant setting, the ATAC (Arimidex and Tamoxifen, Alone and in Combination) trial compares anastrozole, either alone or in combination with tamoxifen, with tamoxifen alone, as first-line endocrine therapy for post-menopausal patients.

Letrozole is a third-generation aromatase inhibitor which does not significantly suppress aldosterone or cortisol production. The results of a phase III trial comparing letrozole (at two different doses) with megestrol acetate in post-menopausal women with advanced disease, have recently been published (Dombernowsky et al. 1998). In the 551 patients randomised, there was a statistically significantly higher response rate in favour of letrozole, at a dose of $2.5 \mathrm{mg}$, compared with megestrol acetate. Time to progression was also significantly pro-longed. Significantly more patients on megestrol acetate than on either dose of letrozole had serious drug-related adverse or weight gain. A trial involving letrozole as first-line therapy, versus tamoxifen, analogous to the use of anastrozole in the ATAC trial above, is also planned. 
There are currently no clinical data comparing the new generation aromatase inhibitors with one another, although a trial comparing letrozole with anastrozole is planned.

\section{Oestrogen receptor status}

The efficacy of endocrine therapies such as tamoxifen and ovarian suppression, both in the adjuvant treatment of early breast cancer and in treatment of advanced (metastatic) disease is clear. The quality and duration of responses achieved are amongst the best produced by any form of therapy. It is therefore important that patients likely to benefit from endocrine therapy are identified reliably. Equally it is important to identify patients who will not benefit from hormone manipulation, so that therapy can be more effectively targeted, and so that patients who will not benefit can be spared potentially toxic treatments.

The 1998 EBCTCG overview of tamoxifen therapy has highlighted the importance of hormone receptor measurement as a determinant of response to tamoxifen therapy. The 1992 overview had already pointed to a much smaller benefit from tamoxifen therapy in women whose tumours were 'ER-poor', and that variability in the methodology of ER assays between trials must inevitably include some false negatives i.e. some of the responses seen in 'ER-poor' patients might, in fact, be in women who are actually ER-positive, but whose ER status was measured by insufficiently sensitive methods. In other words, the apparent benefit in ER-poor patients might be an over-estimate.

The 1998 EBCTCG overview takes the disparity in response between ER-positive and ER-poor tumours further. Its results indicate that there is no clear evidence of benefit in women whose tumours are 'ERpoor', with no apparent effect of 5 years' tamoxifen on recurrence or mortality. Although the authors emphasise the need for further research into the use of tamoxifen in ER-poor tumours, an argument can thus be made for not treating the ER-poor group with tamoxifen. On this basis, a false negative ER assay, resulting in tamoxifen therapy being inappropriately withheld, is potentially seriously dis-advantageous, as even those with small amounts of ER may benefit from tamoxifen. Thus, if the decision to administer tamoxifen is to be based upon ER assays, it is literally vital that the assay be as sensitive as possible.

Early ER assays involved a biochemical method, the dextran-coated charcoal assay (DCCA), requiring separation of cytosol from homogenised tumour preparations. This assay can produce an over-estimate of the ER-poor group, if non-representative areas of the tumour are used. Subsequently, ER assays have been developed which involve immuno-histochemical, rather than biochemical, assessment of ER content. These ER immunohistochemical assays (ERICAs) can be performed on formalin-fixed tumour slices, allowing confirmation that ERs are actually present on cells with a malignant phenotype. Although there has been considerable debate as to the relative merits of the two types of assay, ERICAs are becoming increasingly accepted as the method of choice for determination of ER status. Although criticism has been levelled at ERICA results, on the grounds that results are only semi-quantitative, involving subjective assessments of staining, Barnes et al. (1996) demonstrated that any one of six different scoring systems produced superior results to a ligand-binding cytosol assay (performed many years previously on the same tissue samples) when used to predict clinical outcome in women with metastatic breast cancer. Alberts et al. (1996) compared the ER status of 316 women entered into two adjuvant chemotherapy trials, as assayed by both DCCA and ERICA methods, finding a $21 \%$ discordance rate between the two types of assay, particularly in pre-menopausal women, and concluded that immuno-histo-chemistry may more accurately reflect the true ER status of malignant cells. In the light of the EBCTCG 1998 overview, therefore, the ERICA assay may be of importance in accurately determining those most likely to benefit from hormonal therapies.

\section{Hormone replacement therapy and breast cancer}

There are two important, but separate, issues with respect to HRT in patients with breast cancer. The first concerns the use of HRT and the risk of subsequently developing breast cancer, and the second is the use of HRT in women with a personal history of breast cancer.

Several meta-analyses of the published literature up to 1996 (reviewed in Helzlsouer et al. 1996 and Roy et al. 1996) consistently suggested that 'ever use' of hormones was not associated with a significant increase in the risk of breast cancer, and that duration of oestrogen use was only weakly associated with breast cancer. However, the Collaborative Group on Hormonal Factors in Breast Cancer (1997) have now published a meta-analysis of data from 160000 women which incorporates $90 \%$ of the epidemiological evidence pertaining to HRT use and the risk of breast cancer world-wide. They concluded that there was a statistically significant increased risk of breast cancer being diagnosed in women who were currently receiving HRT, or who had ceased using it 1-4 years before diagnosis (relative risk 1.35), that the increase was greater with increasing duration of use (relative risk increase of $2.3 \%$ per year of use, compared with those who had never used HRT), and that 
discontinuation of use resulted in almost complete disappearance of the excess risk within 5 years of discontinuation of HRT.

Of course, the risk of developing cancer, and breast cancer in particular, is only one aspect of HRT. Other factors include the relief of menopausal symptoms and quality of life issues, and long-term effects of HRT such as the possible protection from cardiovascular events and reduced rate of bone mineral loss over time and the consequent potential reduction in osteoporotic fractures. Therefore, any risk of breast cancer must be balanced by the expected benefit in any individual.

The use of HRT in women with a personal history of breast cancer remains controversial. Traditionally it has been thought inappropriate for such individuals to receive HRT, predominantly due to concerns that such therapy may increase their risk of developing a recurrence of their breast cancer. These concerns are based on a number of issues: the likelihood that oestrogen is implicated in the aetiology of breast cancer; the fact that a significant proportion of breast cancers have receptors for oestrogen, which might therefore stimulate proliferation of tumour; the continuing concerns regarding the use of HRT and an individual's subsequent risk of developing breast cancer; and that ovarian ablation and anti-oestrogenic drugs are effective in both adjuvant treatment and the treatment of metastatic disease.

However, others have argued against prohibiting the use of HRT in women with a previously treated breast cancer on a number of grounds, particularly the lack of definite clinical evidence that the use of HRT results in a worse outcome (the Collaborative Group on Hormonal Factors in Breast Cancer (1997) states that, 'without follow-up information, it is not possible to know whether or not long-term use of HRT affects mortality from breast cancer'), and that such treatment may greatly improve the quality of life of a proportion of women with breast cancer whose menopausal symptoms cannot be well controlled by other means.

Given that, with current screening programmes, more women are being diagnosed and treated with very early breast cancer with a very good natural history, that (in line with the recently published recommendations of the NSABP-B20 trial investigators, who demonstrated a reduced risk of breast cancer recurrence in node-negative, oestrogen receptor-positive breast cancer patients treated with chemotherapy and tamoxifen, compared with tamoxifen alone - Fisher et al. 1997) adjuvant chemotherapy is increasingly being used in women with node-negative as well as node-positive patients, and that a number of recent reports of the use of HRT in such patients, while not demonstrating definite safety, have not suggested a significant detrimental effect with respect to recurrent carcinoma, a number of investigators have begun formally to address this question. A recent review (Roy et al. 1996) discussed three published series of women with breast cancer who had received HRT for the relief of menopausal symptoms (Stoll \& Parbhoo 1988, DiSaia et al. 1993, Powles et al. 1993), and two case control studies (Wile et al. 1993, Cobleigh et al. 1994). Although these small reports suggested that HRT can control menopausal symptoms in women with breast cancer without any dramatic increase in breast cancer, the authors concluded that the reports, in which the total number of women is small and the follow-up relatively short, illustrated the fact that data regarding HRT in women with breast cancer are scarce and that any observations must be viewed as preliminary until larger randomised studies have been performed.

Such trials, and trials of HRT in combination with other therapies, are currently being proposed (Roy et al. 1996, Holmberg 1996). An example is a Scandinavian Breast Group, International Breast Cancer Study Group and Cancer Research Campaign collaboration which has proposed a study protocol randomising symptomatic women with a personal history of breast cancer and who have completed other treatment, to HRT or to best available symptomatic treatment without hormones.

\section{Current recommendations and likely future directions}

Hormonal therapies have long been accepted to be amongst the most successful treatments for breast cancer. Tamoxifen has a clearly proven role in the adjuvant treatment of early breast cancer and in the management of metastatic breast malignancies in both pre- and postmenopausal ER-positive women. Some issues related to the optimum use of tamoxifen, particularly the duration of therapy, and its role in ER-poor tumours, remain to be resolved, and mature data on the use of tamoxifen in prevention are awaited. For the time being, tamoxifen remains the drug of choice in first-line endocrine management, although newer anti-oestrogens, potentially with improved therapeutic indices, are being developed. Aromatase inhibitors have now largely replaced progestogens as second-line therapy in post-menopausal women, and some are sufficiently promising that they are now undergoing trials against tamoxifen as first-line therapy in both early and advanced breast cancer. The benefit of ovarian suppression in pre-menopausal women is now clear, but further data are necessary to determine its exact role in combination with other adjuvant therapies. Active participation in on-going trials of both existing and emerging hormone therapies in breast cancer is strongly encouraged, and the outcome of these trials eagerly awaited. 


\section{References}

Alberts SR, Ingle JN, Roche PR, Cha SS, Wold LE, Farr GH Jr, Krook JE \& Wieand HS 1996 Comparison of estrogen receptor determinations by a biochemical ligand binding assay and immunohistochemical staining with monoclonal antibody, ERIDS in female with lymph node positive breast carcinoma entered on two prospective clinical trials. Cancer 78 764-772.

Assikis VJ, Neven P, Jordan VC \& Vergote I 1996 A realistic clinical perspective of tamoxifen and endometrial carcinogenesis. European Journal of Cancer 32A 1464-1467.

Barakat RB 1996 Tamoxifen and endometrial cancer. American Society of Clinical Oncology (Educational Book) 177-181.

Barnes DM, Harris WH, Smith P, Millis RR \& Rubens RD 1996 Immunohistochemical determination of oestrogen receptor: comparison of different methods of assessment of staining and correlation with clinical outcome of breast cancer patients. British Journal of Cancer 74 1445-1451.

Beatson GT 1896 On the treatment of inoperable cases of carcinoma of the mamma. Suggestions for a new method of treatment with illustrative cases. Lancet ii 104-107.

Breast Cancer Trials Committee, Scottish Cancer Trials Office 1987 Adjuvant tamoxifen in the management of operable breast cancer: the Scottish trial. Lancet ii 171-175.

Buzdar A, Jonat W, Howell A, Jones SE, Blomquist C, Vogel CL, Eurmann W, Wolter JM, Azale M, Webster A \& Plourde for the Arimidex study group 1996 Anastrazole, a potent and selective aromatase inhibitor, versus megestrol acetate in postmenopausal women with advanced breast cancer: results of overview analysis of two phase III trials. Journal of Clinical Oncology 14 2000-2011.

Clinical Trials Service, Radcliffe Infirmary, Oxford. Adjuvant Tamoxifen Longer Against Shorter (ATLAS) Protocol, April 1995. ATLAS Office, Clinical Trials Service Unit, Radcliffe Infirmary, Oxford OX2 6HE, UK.

Cobleigh MA, Berris RF, Bush T, Davidson NE, Robert NJ, Sparano JA, Tormey DC \& Wood DC 1994 Oestrogen replacement therapy in breast cancer survivors. A time for change. Journal of the American Medical Society 272 540545.

Cocconi G 1996 New generation of aromatase inhibitors: prospects of a major advantage for the patient. Annals of Oncology 7 433-437.

Collaborative Group on Hormonal Factors in Breast Cancer 1997 Breast cancer and hormone replacement therapy: collaborative reanalysis of data from 51 epidemiological studies of 52705 women with breast cancer and 108411 women without breast cancer. Lancet 350 1047-1059.

Current Trials Working Party of the Cancer Research Campaign Breast Cancer Trials Group 1996 Preliminary results from the Cancer Research Campaign Trial evaluating tamoxifen duration in women aged fifty years or older with breast cancer. Journal of the National Cancer Institute 88 1834-1839.

Curtis RE, Boice JD, Shriner DA, Hankey BF \& Fraumeni JF 1996 Second cancers after adjuvant tamoxifen therapy for breast cancer (brief communication). Journal of the National Cancer Institute $\mathbf{8 8} 832-834$.
DiSaia PJ, Odicino F, Grosen EA, Cowan B, Pecorelli S \& Wile AG 1993 Hormone-replacement therapy in breast cancer (letter). Lancet 3421232.

Dombernowsky P, Smith I, Falkson G, Leonard R, Panasci L, Bellmunt J, Gardin G, Gudgeon A, Morgan M, Fornasiero A, Hoffman W, Michel J, Hatschek T, Tjabbes T, Chaudri HA, Hornberger U \& Trunet PF 1998 Letrozole, a new oral aromatase inhibitor for advanced breast cancer: double-blind randomized trial showing a dose effect and improved efficacy and tolerability compared with megestrol acetate. Journal of Clinical Oncology 16 453-461.

Early Breast Cancer Trialists' Collaborative Group 1990 Treatment of Early Breast Cancer, vol 1, Worldwide Evidence 1985-1990. Oxford: Oxford University Press.

Early Breast Cancer Trialists' Collaborative Group 1992a Systemic treatment of early breast cancer by hormonal, cytotoxic, or immune therapy. 133 randomised trials involving 31000 recurrences and 24000 deaths among 75000 women (Part 1). Lancet 339 1-15.

Early Breast Cancer Trialists' Collaborative Group $1992 b$ Systemic treatment of early breast cancer by hormonal, cytotoxic, or immune therapy. 133 randomised trials involving 31000 recurrences and 24000 deaths among 75000 women (Part 2). Lancet 339 71-85.

Early Breast Cancer Trialists' Collaborative Group 1996 Ovarian ablation in early breast cancer: overview of the randomised trials. Lancet 348 1189-1196.

Early Breast Cancer Trialists' Collaborative Group 1998 Tamoxifen for early breast cancer: an overview of the randomised trials. Lancet (In Press).

Elwood JM \& Boyes 1980 Clinical and pathological features and survival of endometrial cancer patients in relation to prior use of oestrogens. Gynecologic Oncology 10 173-187.

Falkson CI \& Falkson HC 1996 A randomised study of CGS 16949A (fadrozole) versus tamoxifen in previously untreated postmenopausal patients with metastatic breast cancer. Annals of Oncology 7 465-469.

Fisher B \& Redmond C 1992 Systemic therapy in node-negative patients: updated findings from NSABP clinical trials. National Surgical Adjuvant Breast and Bowel Project. Monographs of the National Cancer Institute 11 105-116.

Fisher B, Constantino J, Redmond C, Poisson R, Bowman D, Couture J, Dimitrov NV, Wolmark N, Wickerham DL, Fisher ER et al. 1989 A randomised clinical trial evaluating tamoxifen in the treatment of patients with node-negative breast cancer who have oestrogen-receptor positive tumours. New England Journal of Medicine 320 479-484.

Fisher B, Constantino JP, Redmond CK, Fisher ER, Wickerham DL \& Cronin CK 1994 Endometrial cancer in tamoxifen-treated breast cancer patients: findings from the National Surgical Adjuvant Breast and Bowel (NSABP) B-14. Journal of the National Cancer Institute 86 527-537.

Fisher B, Dignam J, Bryant J, DeCillis A, Wickerham DL, Wolmark N, Costantino J, Redmond C, Fisher ER, Bowman DM, Deschênes L, Dimitrov NV, Margolese RG, Riobidoux A, Shibata H, Terz J, Paterson AHG, Feldman MI, Farrar W, Evans J \& Lickley HL 1996 Five versus more than five years of tamoxifen therapy for breast cancer patients with 
negative lymph nodes and oestrogen receptor-positive tumours. Journal of the National Cancer Institute $\mathbf{8 8} 1529$ 1542 .

Fisher B, Dignam J, Wolmark N, DeCillis A, Emir, B, Wickerham DL, Bryant J, Dimitrov NV, Abramson N, Atkins JN, Shibata H, Deschenes L \& Margolese RG 1997 Tamoxifen and chemotherapy for lymph node-negative, estrogen receptor-positive breast cancer. Journal of the National Cancer Institute 89 1673-1682.

Fornander T, Rutqvist LE, Cedermark B, Glas U, Mattsson A, Silfversward C, Skoog L, Somell A, Theve T, Wilking N, Ledermark B et al. 1989 Adjuvant tamoxifen in early breast cancer: occurrence of new primary cancers. Lancet 1 117-120.

Goss PE \& Gwyn KM 1994 Current perspectives on aromatase inhibitors in breast cancer. Journal of Clinical Oncology 12 2460-2470.

Gradishar WJ \& Jordan VC 1997 Clinical potential of new antiestrogens. Journal of Clinical Oncology 15 840-852.

Grady D, Gebretsadik T, Kerlikowske K, Ermster V \& Petitti D 1995 Hormone replacement therapy and endometrial cancer: a meta-analysis. Obstetrics and Gynaecology 85 304-313.

Greaves P, Goonetilleke R, Nunn G, Topham J \& Orton T 1993 Two-year carcinogenicity study of tamoxifen in Alderley Park Wiston-derived rats. Cancer Research 53 3919-3924.

Hard GC, Iatropoulos MJ, Jordan K, Radi L, Kaltenberg OP, Imondi AR \& Williams GM 1993 Major differences in the hepato-carcinogenicity and DNA adduct forming ability between toremifen and tamoxifen in female $\mathrm{Crl}: \mathrm{CD}(\mathrm{BR})$ rats. Cancer Research 53 4534-4541.

Hayes DF, Van Zyl JA, Hacking A, Goldhals L, Bezwoda WR, Mailliard JA, Jones SE, Vogel CL, Berris RF, Shemano I et al. 1995 Randomized comparison of tamoxifen and two separate doses of toremifine in post-menopausal patients with metastatic breast cancer. Journal of Clinical Oncology 113 2556-2566

Helzlsouer KJ 1996 Hormone replacement therapy and the risk of developing breast cancer. American Society of Clinical Oncology (Educational Book) 184-186.

Holmberg L 1996 Hormonal replacement therapy for women with a personal history of breast cancer? Annals of Oncology 7 655-656.

Howell A, DeFriend DJ, Robertson JFR, Blamey RW, Anderson L, Anderson E, Sutcliffe FA \& Walton P $1996 a$ Pharmacokinetics, pharmacological and anti-tumour effects of the specific anti-oestrogen ICI 182780 in women with advanced breast cancer. British Journal of Cancer 74 300-308

Howell A, Downey S \& Anderson E 1996b New endocrine therapies for breast cancer. European Journal of Cancer 32A 576-588.

Huggins C \& Dao T 1953 Adrenalectomy and oophorectomy in the treatment of advanced carcinoma of the breast. Journal of the American Medical Association 151 1388-1394.

Jordan VC 1995a What if tamoxifen (ICI 46,474) had been found to produce liver tumours in 1973? A personal perspective. Annals of Oncology 6 29-34.

Jordan VC 1995b Tamoxifen and tumorigenicity: a predictable concern (editorial). Journal of the National Cancer Institute 87 623-626.
Killackey MA, Hakes TB \& Pierce VK 1985 Endometrial adenocarcinoma in breast cancer patients receiving antioestrogens. Cancer Treatment and Research 69 237-238

Kristensen B, Ejlersten B, Dalgaurd P, Larsen L, Holmegaard SN, Transbol I \& Mouridsen HT 1994 Tamoxifen and bone metabolism in post-menopausal low-risk breast cancer patients: a randomised study. Journal of Clinical Oncology 12 992-997.

Love RR, Mazess RB, Toumey DC, Barden HS, Newcomb PA \& Jordan VC 1988 Bone mineral density in women treated with adjuvant tamoxifen for at least two years. Breast Cancer Research and Treatment 12 297-301.

Love RR, Newcomb PA, Wiebe DA, Surawicz TS, Jordan VC, Carbone PP \& DeMets DL 1990 Effects of tamoxifen therapy on lipid and lipoprotein levels in post-menopausal patients with node-negative breast cancer. Journal of the National Cancer Institute 82 1327-1332.

Luft R \& Olivecrona H 1953 Experience with hypophysectomy in man. Journal of Neurosurgery 10 301-316.

McDonald CC \& Stewart HJ 1991 Fatal myocardial infarction in the Scottish adjuvant tamoxifen trial. The Scottish Breast Cancer Committee. British Medical Journal 303 435-437.

McDonald CC, Alexander FE, Whyte BW, Forrest AP \& Stewart HJ 1995 Cardiac and vascular morbidity in women receiving adjuvant tamoxifen for breast cancer in a randomised trial. The Scottish Cancer Trials Breast Group. British Medical Journal 311 977-980.

National Cancer Institute Clinical Announcement. Adjuvant therapy of breast cancer - tamoxifen update. 30th November 1995. Bethesda, MD: National Institutes of Health.

Nayfield SG \& Gorin MB 1996 Tamoxifen-associated eye disease: a review. Journal of Clinical Oncology 14 1018-1026.

Nayfield SG, Karp JE, Ford LG, Dorr FA \& Kramer BS 1991 Potential role of tamoxifen in prevention of breast cancer. Journal of the National Cancer Institute 83 1450-1459.

Peto R 1996 Five years of tamoxifen or more? Journal of the National Cancer Institute 88 1791-1793.

Powles TJ 1996 Tamoxifen, bone, lipids and gynecology. American Society of Clinical Oncology (Educational Book) 174-176.

Powles TJ, Hickish T, Casey S \& O'Brian M 1993 Hormone replacement after breast cancer. Lancet 342 60-61.

Powles TJ, Hickish T, Kanis JA, Tidy A \& Ashley S 1996 Effect of tamoxifen on bone mineral density measured by dualenergy X-ray absorptiometry in healthy pre-menopausal and post-menopausal women. Journal of Clinical Oncology 14 78-84.

Roy JA, Sawka CA \& Pritchard KI 1996 Hormone replacement therapy in women with breast cancer: do the risks outweigh the benefits? Journal of Clinical Oncology 14 997-1006.

Rutqvist LE, Mattson A for the Stockholm Breast Cancer Study Group 1993 Cardiac and thrombo-embolic morbidity among post-menopausal women with early stage breast cancer in a randomised trial of adjuvant tamoxifen. Journal of the National Cancer Institute 85 1398-1406.

Rutqvist LE, Johansson H, Signomklao T, Johansson V, Fornander T \& Wilking N 1995 Adjuvant tamoxifen therapy for early stage breast cancer and second primary 
Wyld et al.: Endocrine aspects of the clinical management of breast cancer

malignancies. Journal of the National Cancer Institute $\mathbf{8 7}$ 645-651.

Santen RJ, Worgul TJ, Samoljik E, Interrante A, Boucher AE, Lipton AM, Harvey HA, White DS, Smart E, Cox C \& Wells S 1981 A randomised trial comparing surgical adrenalectomy with aminoglutethimide plus hydrocortisone in women with advanced breast cancer. New England Journal of Medicine 305 545-551.

Scottish Cancer Trials Breast Group and ICRF Breast Unit, Guy's Hospital, London 1993 Adjuvant ovarian ablation versus CMF chemotherapy in premenopausal women with pathological stage II breast carcinoma: the Scottish trial. Lancet 341 1293-1298.

Simon R 1995 Discovering the truth about tamoxifen: problems of multiplicity in statisticalevaluation of biomedical data. Journal of the National Cancer Institute $87627-$ 629.

Stein RC, Coombes RC \& Howell A 1995 The basis of hormonal therapy of cancer. In Oxford Textbook of Oncology, pp 629648. Eds M Peckham, H Pinedo \& U Veronesi. Oxford: Oxford University Press.

Stewart HJ, Forrest AP, Everington D, McDonald CC, Dewar JA, Hawkins RA, Prescott RJ \& George WD 1996 Randomised comparison of 5 years of adjuvant tamoxifen with continuous therapy for operable breast cancer. The
Scottish Cancer Trials Breast Group. British Journal of

Cancer 74 297-299.

Stoll BA \& Parbhoo S 1988 Treatment of menopausal symptoms in breast cancer patients. Lancet 1 1278-1279.

Swedish Breast Cancer Co-operative Group 1996 Randomised trial of two versus five years of adjuvant tamoxifen for postmenopausal early stage breast cancer. Journal of the National Cancer Institute 88 1543-1549.

Thurlimann B, Beretta K, Bacchi M, Castiglione-Gertsch M, Goldhirsch A, Jungi WF, Cavalli F, Senn H-J, Fey M \& Lohnert $\mathrm{T}$ for the Swiss Group for Clinical Cancer Research (SAKK) 1996 First-line fadrozole HCl (CGS 16949A) versus tamoxifen in postmenopausal women with advanced breast cancer. Annals of Oncology 7 471-479.

Tormey DC, Gray R \& Fakson HC for the Eastern Co-operative Oncology Group 1996 Post-chemotherapy adjuvant tamoxifen therapy beyond 5 years in patients with lymph node-positive breast cancer. Journal of the National Cancer Institute $\mathbf{8 8}$ 1828-1833.

Wile AG, Opfell RW \& Margileth DA 1993 Hormone replacement therapy in previously treated breast cancer patients. American Journal of Surgery 165 372-375.

Williams GM, Iatropoulos MJ, Djordjevic MV \& Kaltenberg OP 1993 The triphenylethylene drug tamoxifen is a strong liver carcinogen in the rat. Carcinogenesis 14 315-317. 